\title{
Der Uebergang von Chloroform und Salicylsäure in die Placenta,
}

nebst Bemerkungen über den Icterus neonatorum.

Von

P. Zweifel.

Meine ersten Versuche über diesen Gegenstand wurden mit dem Urin von Neugeborenen begonnen, deren Mütter während der Geburt kürzere oder längere Zeit chloroformirt worden waren. Es gab der Urin dieser Kinder, nach Elimination der Harnsäure, bei der Trommer'schen Probe eine Reduction des Kupfersulphats. Im Harne von normal geborenen Kindern trat in 25 darauf untersuchten Fällen bei ganz gleicher Behandlung niemals eine Reduction ${ }^{1}$ ) ein. Einen Einfluss des Chloroforms darin zu sehen, war um so naheliegender, als die Annahme nichts Befremdendes haben konnte, dass das Chloroform als Gas aus dem Kreislaufe der Mutter in denjenigen des Kindes ïbergehe, denn ein Uebergang von Sauerstoff musste bei der bestehenden Lehre von der fötalen Athmung als sicher angenommen werden. Freilich konnte die Placentarrespiration, die doch durch die grosse Affinität des Sauerstoffes zu den rothen Blutkörperchen eine ganz andere Basis hat, blos eine Wahrscheinlichkeit für den Uebergang von Chloroform abgeben. In der oben citirten Abhandlung ist die Angabe gemacht, dass die Urinuntersuchungen vorgenommen wurden, weil einmal in der Exspirationsluft des Kindes Chloroformgeruch wahrzunehmen war.

1) Siehe „Einfluss der Chloroforminarkose Kreissender auf den Fötus“. Berliner klinische Wochenschrift 1874, Nr. 21. 
Im Urin Chloroformirter war von Reynoso, Sabarth u. A. eine reducirende Substanz aufgefunden und daraus der Schluss gezogen worden, dass dies ein Stoffwechselproduct der Narkose sei. Die reducirende Substanz, die ich im Urin des Kindes finden konnte, geht entweder fertig gebildet aus dem Blute der Mutter in die Placenta über oder wird im Kreislaufe des Kindes aus übergegangenem Chloroform erst gebildet. Die Entscheidung zwischen diesen beiden Möglichkeiten war nur durch eine Untersuchung des Fötusblutes auf Chloroformgehalt zu erreichen.

Hierzu benutzte ich eine Placenta, welche von den anhaftenden Blutklumpen gut gereinigt, dann rasch verkleinert wurde und nun zur Destillation kam. Um die Chloroformdämpfe rascher überzuführen, wurde ein Luftstrom durch den betreffenden Glaskolben geleitet, die Dämpfe in einer glühenden Kaliglasröhre zersetzt und die entstandenen Producte in eine Lösung von Silbernitrat übergeführt: Die Bildung von Chlorsilber bewies in diesem Falle mit aller Evidenz das Vorhandensein von Chloroform in der Placenta und berechtigte zu dem Schlusse, dass das Chloroform als solches in das, Fötalblut übergehe und dort die reducirende Substanz bilde.

Eine Publication von Hüter, über die Wirksamkeit des Chloroforms bei geburtshülf lichen Operationen 1) enthält Mittheilungen von Versuchen, welche Hüter mit den Herren Zwenger und Nasse ausgeführt hatte, um das Chloroform im Fötalblute nachzuweisen. Es wurde das Blut aus dem Nabelstrange ausgedrückt, dasselbe der Destillation. unterworfen, die Chloroformdämpfe nach der Methode von Ragsky in einem glühenden Glasrohre zersetzt und dann an einem Papierstreifen, der mit Amylum und Jodkalium befeuchtet worden war, vorbeigeleitet. Der eine Versuch misslang, der zweite hatte ein positives Resultat; aber der Verfasser macht 1. c. S. 390 die Angabe, dass er zu dem aus dem Nabelstrange gewonnenen Blute (4 Gramm) noch dasjenige von der äusseren Fläche der Placenta hinzugenommen habe. Mit dieser Quantität betrug die untersuchte Blutmenge circa 15 Gramm; aber damit hatte der Versuch auch das Entscheidende verloren, indem das nachträglich hinzugefügte Blut wesentlich mütterliches war.

1) Neue Zeitschrift für Geburtskunde, Bd, 27. 
Neulich publicirte Fehling ${ }^{1}$ ) Versuche, bei denen trächtige Thiere mit Curare und Chloroform vergiftet wurden. Mit dem Chloroform wurde nur ein Versuch bei einem Kaninchén gemacht: 18 Minuten nach eingetretener tiefer Narkose wurde der Uterus eröffnet. Die Jungen bewegten sich noch und machten-spontane Athembewegungen. Dieser eine negative Versuch beweist aber selbstverständlich gar nichts gegen die oben begründete Ansicht, dass das Chloroform in das Fötalblut übergehe. Auch die Kinder, welche auf den Einfluss des übergegangenen Chloroform untersucht worden, bewegten sich spontan nach der Geburt und begannen in regelmässiger Weise ihre Athembewegungen, währead ihre Mütter ununterbrochen in tiefster Narkose gehalten wurden. Es lässt dies höchstens darauf schliessen, dass die Chloroformwirkung auf den Fötus relativ viel geringer sei und wahrscheinlich dem entsprechend auch das fötale Blut relativ viel weniger Chloroform enthalte als das mütterliche.

Es sind diese Beziehungen hervorzuheben, da andere Autoren $^{2}$ ) die Fehling'schen Versuche als beweisend für ihre subjective Ansicht citirten, dass keine Arzneistoffe den Fötus beeinflussen können, eine Ansicht, die Fehling selbst nicht daraus geschlossen hatte.

Trotzdem ich die Placenta nach Möglichkeit von mütterlichem Blute gereinigt hatte, konnte ich die schwache Seite des Destillationsversuches, dass nämlich noch kleine Quantitäten mütterlichen Blutes mit verwendet worden, nicht unbedingt bestreiten, und das gab die Veranlassung, neue Versuche anzustellen.

Um dem Einwande zu entgehen, dass mütterliches Blut mit benutzt werde, wurde blos das durch Ausdrücken der Placenta gewonnene Blut untersucht. Die so aufgefangene Quantität betrug zuweilen circa $30 \mathrm{Ccm}$, die einfache Destillation in einem Glaskölbchen hatte aber besondere Schwierigkeiten. Das Blut kam auf dem Wasserbade bald zur Gerinnung, und von da an war die Destillation ohne Erfolg, man konnte erhitzen, so lange man wollte. Auch das Durchleiten eines Luftstromes half nur unvoll-

1) Zur Lehre vom Stoffwechsel zwischen Mutter und Kind. Dieses Archiv, Bd. IX, S. 313 u. ff.

2), Vergl. Centralblatt für Gynäkologie 187\%, S. 129. -- Munde, Barker, Peaslee, Gillette, Thomas, Ueber den Einfluss von Medicamenten auf den Fötus. 
kommen, und es war ohne Weiteres bemerkbar, dass auf diese Weise immer nur der kleinste Theil Chloroform zur Destillation kommen könne. Ich versuchte nun, das Chloroform mit Alkohol auszuziehen und von diesem abzudestilliren.

$\mathrm{Da}$ es bei diesen ersten Versuchen blos darauf ankam, überhaupt Chloroform zu finden, ohne zu gleicher Zeit die Quantität zu bestimmen, so machte ich mit dem Destillat zuweilen rasch die Hoffmann'sche Carbylaminreaction. ') Das Chloroform bildet mit alkoholischer Kalilauge und einem Monamin, z. B. dem Anilin (Phenylamin) gekocht, Phenylcarbylamin, das durch seinen penetranten, höchst charakteristischen Geruch den Nachweis mit aller Präcision gestattet. Die Umsetzung geschieht nach der Formel :

$$
\mathrm{CHCl}_{3}+\mathrm{C}_{6} \mathrm{H}_{5} \cdot \mathrm{NH}_{2}+3 \mathrm{KOH}=\mathrm{C}_{6} \mathrm{H}_{5} \mathrm{NC}+3 \mathrm{~K} \mathrm{Cl}+3 \mathrm{H}_{2} \mathrm{O} \text {. }
$$

In mehreren Versuchen, die ich im Laufe des Sommersemesters 1876 nach Erscheinen der Fehling'schen Arbeit ausgeführt habe, bekam ich mit den ersten Tropfen des Destillates die Reaction mit aller Bestimmtheit.

Für allfällige Controlversuche muss ich darauf aufmerksam machen, dass ja nicht der extrahirende Alkohol ohne alle Blutgerinnsel abgegossen und zur Destillation gebraucht werde, sonst ist mit allergrösster Wahrscheinlichleeit ein negatives Resultat zu erwarten. Selbstverständlich müssen die einzelnen Proceduren ganz rasch, in möglichst kurzer Frist nach dem Auffangen des Blutes ausgeführt werden.

1. Versuch. Das Chloroform kam wegen excessiv heftiger Schmerzen zur Anwendung, eine Stunde später wurde wegen engen Beckens die Wendung gemacht. Hierzu war die tiefste Narkose nothwendig. An die Wendung wurde die Extraction angeschlossen. Die Dauer der Narkose während der Operation war eine Stunde.

Das Blut der Placenta wurde gleich gesammelt und in der angegebenen Weise untersucht. Der Carbylamingeruch war penetrant.

2. Versuch. Die Geburt begann gegen Abend. Ende der I. P. $10 \mathrm{Uhr}$, der II. P. 11/4 Uhr. Es musste im Beckenausgange die Zange angelegt werden. Chloroformirt wurde ungefähr eine Viertelstunde. Im Blate deutliche Chloroformreaction.

3. Versuch. Acht bis neun Minuten dauernde Chloroformnarkose. Durch einen Irrthum wurde Aether statt Alkohol auf das

1) Monatsberichte der Academie der Wissenschaften, Berlin 1870 . 
Blut aufgegossen; nach dem Verjagen des Aethers wurde die Reaction mit dem Rückstande gemacht. Carbylamingeruch nicht deutlich.

4. Versuch. Chloroformirt zur Anlegung der Zange circa 20 Minuten lang. Es wurden $39,93 \mathrm{Gm}$. Blut aufgefangen. Sehr starker Carbylamingeruch.

5. Versuch. Zangenoperation. Chloroformirt wurde circa $\mathbf{1 5 - 2 0}$ Minuten. Carbylamingeruch sehr deutlich.

6. Versuch. Zangenoperation. Chloroformirt von $1 / 210-12$ Ubr. $20 \mathrm{Gm}$. Blut gewonnen; starke Carbylaminreaction.

7. Versuch. Chloroform von $5 \mathrm{Uhr} 20 \mathrm{Min}$. bis $6 \mathrm{Uhr} 20 \mathrm{Min}$. 7 Uhr Beginn der Zangenoperation und circa $1 / 2$ Stunde lang eine starke Narkose. Nach der Geburt gleich $50 \mathrm{Gm}$. Blut ausgedrückt und wie gewöhnlich behandelt. Sehr deutliche Carbylaminreaction.

Diese Versuche beweisen mit Evidenz, dass das Chloroform in das Blut des Fötus übergeht.

Gerade während der Abfassung dieser Arbeit kam mir eine neue Publication von Fehling in diesem Archiv, Band XI, zu Gesicht. Der Verf. giebt auf S. 554 einen Versuch nach meiner Methode an, bei welchem er ebenfalls eine starke Carbylaminreaction bekam. Es hat sich also dieser Forscher nunmehr selbst von der Richtigkeit meiner Angaben überzeugt, gegen welche er. in Hamburg opponirt hatte. In der 49. Versammlung deutscher Naturforscher und Aerzte in Hamburg hatte ich über solche Versuche schon referirt und damals die Absicht ausgesprochen, die Stoffwechselverhältnisse zwischen Mutter und Fötus noch weiter zu verfolgen, indem für die beiden Blutarten quantitative Bestimmungen des Chloroforms ausgeführt werden sollten.

Schmiedeberg 1 ) hatte als beste Bestimmungsmethode des Chloroforms im Blute das Ueberdestilliren nach Einleitung von Kohlenoxydgas, Zersetzen des Destillates im glïhenden Kaliglasrohre und Auffangen der Zersetzungsproducte mit Aetzkalk angegeben. Es hatte nämlich dieser Forscher die Erfahrung machen müssen, dass das Chloroform aufs Hartnäckigste vom Blute zurückgehalten werde. Durch Controlversuche mit Serum konnte eine Zersetzung des Chloroforms durch die Alcalescenz des Blutes ausgeschlossen werden. Während bei den Destillationen von Blut die abgewogene Chloroformmenge nur mit grossen Ver-

1) Ueber die quantitative Bestimmung des Chloroforms im Blute, Dorpat 1866 , 
lusten wiedergefunden werden kounte, ging das Chloroform aus dem Serum ohne nennenswerthen Verlust über.

Daraus schloss Schmiedeberg, dass das Chloroform eine Verbindung mit den Blutkörperchen eingehe, ähnlich wie der Sauerstoff, welche Verbindung durch das. Einleiten von Gasen, die eine noch grössere Affinität zum Blute hätten, gelöst werden könnte. Dadurch würde das Chloroform frei und ohne Verlust aus dem Blute abdestilliren. Die Einleitung von Kohlenoxydgas ergab auch in der That nur noch unbedeutende Verluste an Chlor.

Die Angabe, dass das Chloroform eine Verbindung mit den Blutkörperchen eingehe, veranlasste mich nun zuerst, chloroformhaltiges Blut im Spectralapparate zu untersuchen. Bei genau gleicher Verdünnung zeigte dieses Blut im Vergleiche mit gewöhnlichem keine Veränderung der Absorptionsstreifen des Haemoglobins.

Die Einleitung von Kohlenoxydgas hat nun nach Schmiedeberg's eigenen Angaben sehr grosse Unbequemlichkeiten. Die Hälfte der Versuche misslang durch Entzündung des Gemisches von Kohlenoxyd und atmosphärischer Luft und Explosion desselben mit Zerstörung der Apparate oder doch mit Fortpflanzung der Flamme bis in die Vorlage. Eine solche Methode der quantitativen Bestimmung war meinen Zwecken wenig förderlich. Es musste unbedingt eine Methode den Vorzug verdienen, die das Chloroform, wenn auch nicht absolut genau, so doch mit geringen und gleichmässigen Verlusten bestimmen lässt.

Wenn die Destillation des Chloroforms unter gewissen Cautelen gegen rasche Verdunstung in der Vorlage und im Kühlrohr ausgefïhrt wird, so scheint die Annahme gerechtfertigt, dass der Verlust klein und gleichmässig sei. Im Destillat selbst lässt die oben schon angegebene Umsetzung dẹs Chloroforms in Carbylamin und Chlorkalium respective Chlornatrium eine genaue Bestimmung des Chlorgehaltes zu.

Die ersten Versuche, die zur rationellen Begründung eines solchen Verfahrens angestellt werden mussten, hatten den $Z_{\text {weck, }}$ abgewogene Quantitäten Chloroform zu zersetzen und nachher durch Bestimmung des Chlorgehaltes zu prüfen, ob die Umsetzung ohne Verluste vor sich gegangen sei. Es wurden hierzu kleine Fläschchen von starkem Glase benutzt, bei denen durch eine gut aufgeschliffene Glasplatte ein luftdichter Verschluss herzustellen ist. Das ganze Fläschchen steht in einem Gehäuse von Eisen- 
draht, die Glasplatte wird durch Schrauben fest gegen die Mündung angepresst und zu grösserer Sicherheit des Verschlusses eine feine Kautschuckplatte dazwischen gelegt. Man kann dann mit diesem Apparate die Zersetzung ebenso sicher machen, wie im zugeschmolzenen Glasrohr. Das Chloroform wurde in die schon beschriebenen Gläschen gebracht und mit alkoholischer Natronlauge und Anilin im Wasserbad erhitzt. Das Natriumhydroxyd war ganz rein, durch Verbrennen von Natrium gewonnen.

1. Versuch 0,12 Gm. Chloroform entsprechen 0,112 Gm. Chlor. gefunden wurden $0,106, "$,

2. Versuch $0,08 \mathrm{Gm}$. Chloroform entsprechen $0,071, "$ " gefunden wurden $0,074, "$,

3. Versuch $0,07 \mathrm{Gm}$. $\mathrm{CHCl}_{3}$ entsprechen 0,062 " ", gefunden wurden $0,069, "$,

4. Versuch $1,661 \mathrm{Gm} . \mathrm{CHCl}_{3}$ entsprechen 1,479 " " gefunden wurden $1,476, "$,

Bei den drei ersten Versuchen erklärt sich das Mehr an Chlor durch die zur Verfügung gestandene Waage, die nicht mehr für die Milligramme genau war. Der vierte Versuch war im chemischen Laboratorium des Herrn Prof. Hilger ausgeführt worden.

Eine weitere Reihe von Versuchen ging darauf aus, das Chloroform aus alkoholischen oder wässerigen Lösungen abzudestilliren und hier die Bestimmungen und den Vergleich mit der abgewogenen $\mathrm{Menge} \mathrm{CHCl}_{3} \cdot \mathrm{zu}$ machen. Es waren dabei nur kleine Verluste zu notiren, die jedenfalls von der Verdunstung des Chloroforms in der Vorlage herrührten.

Die dritte Reihe von Destillationen aus Blut, ohne Durchleiten ron Gasen, ergab ganz bedeutende Verluste, welche die Resultate unbrauchbar machten. Es bestätigten diese Versuche die schon ron Schmiedeberg am Rindsblute gemachto Erfahrung, dass das Chloroform aufs Hartnäckigste zurückgehalten werde.

Auf Anrathen des Herrn Prof. Hilger machte ich noch Versuche, das Chloroform mit überhitztem Wasserdampfe aus dem Blute abzudestilliren. Die Zersetzung im glühenden Glasrohre ist hier nicht mehr zulässig, dafür geht aber die Destillation so ausserordentlich rasch vor sich, dass bei guter Kühlung die Verluste sehr gering sind und diese Methode für Destillationen mit BIut sehr zu empfehlen ist. Es 
wurden die Dämpfe in einem Papinianischen Topf durch eine kräftige Gasflamme erzeugt und dann in das Kölbchen mit Blut eingeleitet.

Das Blut kommt stets rasch zur Gerinnung, und es muss deswegen voran mit Wasser verdünnt werden. Die Farbe ändert sich und in Kurzem sieht dasselbe aus wie gekocht.

Wenn auch diese Destillationen mit überhitztem Wasserdampfe viel bessere Resultate ergaben und das Chloroform viel vollkommener aus dem Blute auszuziehen vermochten, kam ich doch zu der Ueberzeugung, dass die Fehlerquellen der Bestimmung noch zu gross seien, um vergleichende Untersuchungen zwischen so geringen Mengen Chloroform anzustellen, wie sie in je 30 Gramm fötalem oder mütterlichem Blute zu erwarten sind.

Um einigermaassen Anhaltspunkte zu bekommen, welche Mengen Chloroform in einer Unze Fötalblut zu erwarten seien, versuchte ich vorerst mit anderen Stoffen, die leichter nachzuweisen sind, Bestimmungen auszuführen. Ich wählte hierfür die Salicylsäure wegen des so bequemen Nachweises.

1) P. bekam $3 / 4$ Stunde vor Vollendung der Geburt 3 Gramm salicylsaures Natron in Oblaten. Im Urin des Kindes war keine Salicylsäure.

2) M. Vier Stunden a. p. 3 Gramm salicylsaures Natron, zwei Stunden später der Urin der Mutter zuerst untersucht, enthält die Salicylsäure. Unmittelbar nach der Geburt wurde das Kind katheterisirt, der Urin enthielt Salicylsäure. Jetzt wurde die Placenta ausgedrïckt, circa $30 \mathrm{Gm}$. Blut gewonnen, dieses mit Alkohol und Aether ausgezogen, das Extractionsmittel verdunstet und nach Wiederauflösen in Wasser verdünntes Eisenchlorid zugesetzt. Es trat eine deutliche roth-violette Färbung ein. Nunmehr wurde eine sehr stark verdünnte Lösung von salicylsaurem Natron hergestellt, deren Gehalt in $5 \mathrm{Ccm}$. 0,000375 betrug. Auch dieser Lösung wurde Eisenchlorid zugesetzt und durch dieses und entsprechende Verdünnung mit Wasser ganz genau dieselbe Färbung bei gleicher Flüssigkeitsschicht erreicht. Durch Vergleichung der beiden Volumina bestimmte ich den Gehaltan salicylsaurem Natron in den $30 \mathrm{Gm}$. Blut z u $0,0005 \mathrm{Gm}$.

3) G. Geburt am 7. Januar 1877. Eine Stunde zehn Minuten vor der Geburt $3 \mathrm{Gm}$. salicylsaures Natron eingenommen. In der Blase des Kindes kein Urin. In Blute, das aus dem Nabelstrange ausgepresst worden, nach der angegebenen Bestimmung 0,000937 $\mathrm{G} \mathrm{m}$. salicylsaures Natron.

4) In diesem Versuche waren jedenfalls weniger als $0,0002 \mathrm{Gm}$. Natrum salicylicum enthalten. 
5) Geburt am 18. Januar 1877. $3 \mathrm{Gm}$. Natrum salicylicum 9 Uhr Morgens gereicht. Geburt vollendet 2 Uhr 15 Minuten. Das gewonnene Blut betrug 23,14 Gm. und enthielt 0,00157 $\mathrm{Gm}$. Natrum salicylicum.

Da die hierbei gefundenen Mengen von salicylsaurem Natron so gering ausfielen, sind die entsprechenden Quantitäten Chlor oform resp. Chlor innerhalb der Fehlergrenzen der Bestim mung, benutze man dazu die Titrirmethode oder die Chlorsilberbestimmung. Dagegen ist nach den Angaben von $\mathrm{Hoffmann}$ die Carbylaminreaction noch genau bis zu einer Verdünnung des Chloroforms von 1:5000-6000. Man wird sich demnach mit dem qualitativen Nachweise durch die $\mathrm{H}$ offmann'sche Reaction begnügen müssen, und es ist ja auch in der That damit erreicht, was wissenschaftlich und praktisch von Interesse ist, nämlich dass das Chloroform als solches in den Kreislauf des Fötus übergeht and die im Urin zu findende reducirende Substanz erst im kindlichen Organismus gebildet wird.

Es lag nahe, bei dem heutigen Stand der Frage vom Uebergange der Salicylsäure in die Placenta dieselbe auch im Fruchtwasser aufzusuchen. Wenn die je in circa $30 \mathrm{Gm}$. Blut gefundene Quantität mit ebensoviel Wasser versetzt wurde, war die violette Färbung kaum mehr zu erkennen. Es konnte also im Fruchtwasser bei der so viel bedeutenderen Verdünnung ohne Einengen gar keine Reaction mehr erwartet werden, selbst wenn der Fötus wiederholt solche Quantitäten Salicylsäure hinein entleert hatte, wie solche unmittelbar nach der Geburt in der Blase zu finden waren. Es ist bekannt, dass Benicke in drei solchen Fällen niemals in dem Fruchtwasser eine Salicylsäurereaction bekommen hatte und wegen dieser negativen Resultate die Theorie Gusserow's, dass der Fötus zeitweise in das Fruchtwasser urinire, anzufechten sich veranlasst sah. Bei seiner Kritik liess er die zwei Fälle von Schauenstein und Spaeth ganz ausser Acht, in denen das Jod nach Jodkaliumgebrauch mit aller Evidenz nachgewiesen worden war. Es existirt in der Literatur noch ein Fall von Righini ${ }^{1}$ ), wo nach Jodoformgebrauch das Jod im Fruchtwasser wieder gefunden worden. Solche positive experimentell chemische Versuche, respective nur ein einziger, der mit allen Cautelen

1) Schmidt's Jahrbücher 1864. 
ausgeführt worden ist, haben mehr Beweiskraft für die Richtigkeit einer Theorie, als ein halbes Dutzend negativer gegen dieselbe beanspruchen können.

Auch Fehling ${ }^{\text {) }}$ wendet sich gegen die Theorie der Harnsecretion des Fötus, aber nur mit hypothetischen Deductionen. Von diesem Autor wird behauptet, dass die Secretion der Nieren nicht möglich sei, weil der Druck im Aortensysteme des Fötus gar nicht genügen würde, um eine Harnsecretion zu bewirken, weil der Druck des Ventrikels gerade ausreiche, das Blut durch die Nabelarterien zu treiben, aber nicht mehr genüge für den erforderlichen Secretionsdruck in den.Nierenarterien!? Diese sehr problematischen Hypothesen und Deductionen sind übrigens durch beweisende Thatsachen bereits widerlegt. Auch die Behauptung Fehling's, dass die Harnabsonderung des Fötus noch durch nichts bewiesen sei, greift leichten Kaufs eine ganze Reihe von Abhandlungen von ausgezeichneten Forschern an. Schwartz ${ }^{2}$ ) hat in seiner Arbeit darauf aufmerksam gemacht, dass alle Versuche, Harnstoff auch in anderen Theilen der Frucht nachzuweisen, vergeblich waren, dagegen hatte er in acht Fällen Harnstoff und Harnsäure im Urin der Kinder gefunden. Hierzu kommt der Fall von Gusserow, wo Wislicenus bei in der Blase vorgefundenem Urin $6,06 \%$ Harnstoff gefunden hatte.

Entscheidend sind aber in dieser Frage die Arbeiten ${ }^{3}$ ), welche den Harnstoff im Fruchtwasser nachgewiesen haben, von Wöhler, Funke $0,38 \%$, Litzmann $0,05 \%$, Majewsky 0,34 und $0,42 \%$, Beale $0,035 \%$, Siewert $0,035 \%$, Winckel $0,42,0,104$ und $0,086 \%$, Guss erow ${ }^{4}$ ) $0,27,0,35,0,14$ und $0,17 \%$, Picard 0,035 und 0,0267, Prochownick ${ }^{5}$ ) in 13 Bestimmungen zwischen 0,0155-0,034\%, im Durchschnitt 0,0204\%. Der Harnstoff wurde sehr oft, von einzelnen regelmässig, als salpetersaurer Harnstoff dargestelit. Ist es gerechtfertigt, diesen so oft wiederholten Untersuchungen gegenüber, wegen einiger

1) Beiträge zur Physiologie des placentaren Stoffverkehrs. Dieses Archiv, XI. Bd., S. 548 u. ff.

2) Die vorzeitigen Athembewegungen.

3) Vergl. Gusserow, dieses Archiv, III. Bd., S. 252.

4) 1. c. S. 253 .

5) Dieses Archiv, XI. Bd, S. 304 u. ff. 
negativer Experımente mit Salicylsäure, die Harnabsonderung des Fötus in Frage zu ziehen?

Der positive Beweis für die zeitweise Harnentleerung des Fötus ist von Runge ${ }^{1}$ ) durch Auffinden der Salicylsäure im Fruchtwasser mit aller Präcision geleistet worden. Im verflossenen Wintersemester waren mir gleiche Versuche misslungen, in denen ich das Natron salicylicum mit absolutem Alkohol auszuziehen versucht hatte. Ich engte die Quantität Fruchtwasser erst auf dem Wasserbade ein, und dabei scheint ein Freiwerden und Verdunsten der Salicylsäure zu Stande gekommen zu sein, denn in anderen seither gemachten Versuchen durch Schütteln mit Aether konnte ich Runge's Mittheilungen vollkommen bestätigen. Es geht auch an, das Fruchtwasser nach einem Zusatze von Natronlauge einzudampfen, dann anzusäuern und mit Aether zu extrahiren.

Der sichere Nachweis einer Urinausscheidung des Fötus führt uns auch eine Erklärungsmöglichkeit für einzelne Fälle von $\mathrm{Hy}-$ dramnion nahe.

Salling e $\mathbf{r}^{2}$ ) hat vier Fälle zusammengestellt, wo bei hydrämischen Müttern Hydramnion entstanden war. Wenn auch die grosse Mehrzahl der Fälle von pạthologischer Vermehrung des Fruchtwassers durch Stauungserscheinungen im Gefässsysteme des Fötus und durch das Persistiren der Vasa propria (Jungbluth) zu erklären ist, so kann andererseits auch das Vorkommen von Hydraemie der Mutter zu Hydramnion führen. Aus dem wässerigen Blute transfundirt mehr Flüssigkeit in das Gefässsystem des Fötus und dieser wird durch die Vermehrung der Blutquantität wiederum mehr Flüssigkeit durch die Nieren absondern können. Damit aber hierdurch ein Hydramnion entstehe, müssen die abnormen Diffusionsverhältnisse dauernd bestehen, weil sonst durch das Verschlucken des Fruchtwassers der Ueberschuss wieder in das mütterliche Blut zurücktreten könnte.

Zu der oben gegebenen Begründung, dass das Chloroform als solches in das Fötalblut übergehe, kann ich noch zwei Thatsachen anführen, die den Uebergang für sich allein wahrscheinlich machen.

1) Centralblatt für Gynäkologie Nr. 5, $18 \mathrm{ff}$.

2) Diss. inaug. (unter Leitung von Frankenhäuser gearbeitet), Zürich 1875 . 
Bei früheren Versuchen mit Secale cornutum mussten öfters Frösche chloroformirt werden, um während der Narcose den Einfluss des Secale respective seiner Salze auf die Gefässe zu sehen. Die Frösche waren in der gewöhnlichen Weise ausgespannt und die Schwimmhaut mit ihren Gefässen zur mikroskopischen Beobachtung ausgebreitet. Wenn die Thiere Chloroform einzuathmen bekamen, sah ich von Zeit zu Zeit helle Kügelchen durch die Gefässe ziehen, die wahrscheinlich aus Chloroform in Gasform bestanden. Bei allen übrigen Beobachtungen der Froschschwimmhaut habe ich sonst nie diese Beobachtung machen können.

Der zweite Punkt, den ich hier erwähnen will, ist der U ebergang des Chloroforms in den Urin der Narcotisirten. $\mathrm{Zu}$ wiederholten Malen hatte ich bei sehr lange dauernden Chloroformnarcosen den Urin mit Anilin und alkoholischer Kalilauge gleich nach dem Katheterisiren gekocht und die deutlichste Carbylaminreaction bekommen, während der von Chloroform freie Harn (in den Controlprüfungen) diese Reaction nicht giebt.

Ich führe die einzelnen Fälle dieses Nachweises nicht an, dafür eine Untersuchung, wo der Urin mit überhitztem Wasserdampfe abdestillirt wurde:

Der Urin nach Vollendung der Narcose (Operation einer Ureterenuterusfistel) aufgefangen und nach der angegebenen Methode abdestillirt. Die Destillation wurde sehr bald unfreiwillig unterbrochen. Im Destillat sehr deutliche Carbylaminreaction und 0,0092 Gramm Chlor. Die zugesetzte alkoholische Natronlauge bestand aus ganz reinem, vollkommen chlorfreiem Natriumhydroxyd.

Wenn das Chloroform die Nieren passirt, so kann es gewiss auch in die fötale Placenta übergehen.

Durch die bis jetzt angeführten Untersuchungen habe ich den Beweis geleistet, dass die im Urin der Kinder zu findende Substanz auf übergegangenes Chloroform bezogen werden kann. Dass aber das Chloroform wirklich zur Bildung dieser Substanz beitrage, wurde durch die fortgesetzte Untersuchung des Urins der Neugeborenen bewiesen.

Wie schon in meiner Mittheilung im Jahre 1874 angegeben worden, fand sich die reducirende Substanz im Alkoholextract des eingedampften Urins ganz regelmässig vor bei Kindern, die während einer Chloroformnarcose ihrer Mutter geboren wurden. Bei 25 Urinproben von normal geborenen Kindern trat bei ganz 
gleicher Behandlung nie eine Reduction ein. Ich habe seit der vorläufigen Mittheilung bei allen Kindern, die dem Einflusse des Chloroforms ausgesetzt gewesen und die ich darauf untersuchen konnte, die reducirende Substanz wiedergefunden. Dass dagegen eine reducirende Substanz (Zucker) im Urine normaler Kinder fehlt, haben auch Bernard ${ }^{1}$ ), William Moore ${ }^{2}$ ) und Parrot und Robin ${ }^{3}$ ) angegeben.

Ueber die Frage, was die reducirende Substanz sei, kann nach den Befunden im Urin der Neugeborenen keine weitere Auskunft gegeben werden. Im Harne Erwachsener haben andere Autoren nach Chloroform eine Reduction bekommen und daraus den Schluss gezogen, dass dieser Urin Zucker enthalte. So ungerechtfertigt es ist, aus der einfachen Reduction des Kupfersulphates auf Zucker zu schliessen, so wiederholen sich solche mangelhafte Untersuchungen doch immerfort und werden darauf hin weitgehende Theorien begründet. Die reducirende Substanz im Urin der Chloroformirten ist mit höchster Wahrscheinlichkeit kein Zucker. Ich verweise hier auf die Untersuchungen von Mering ${ }^{4}$ ), der bei Chloral- und Crotonchloralhydrat im Urin geringe Mengen des dargereichten Stoffes fand, neben einem links drehenden, gechlorten Körper, der in Alkohol löslich war und Kupfersulphatlösung reducirte. Gährungsversuche hatten ein negatives Resultat ergeben. Dass die gleiche Substanz auch im Urin der Kinder sich vorfinde, lässt sich erschliessen aus der Löslichkeit in Alkohol, dem Reductionsvermögen und der linksseitigen Drehung des polarisirten Lichtes.

Bei Erwachsenen kommt das Chloroform zu einem gewissen Theile unverändert in den Urin. Das Findampfen des Urins u. s. w. lässt nun gewiss den Schluss zu, dass die zuletzt der Trommer'schen Probe unterworfene Lösung kein urverändertes Chloroform mehr enthalten könne. Aber es ist nicht einfach von der Hand zu weisen, dass sich die reducirende Substanz erst

1) Bernard (Leçons de physiologie expérimentale 1855, 21. Vorlesung) hatte bei Embryonen aus der früheren Fötalzeit Zucker (Reduction und Linksdrehung im Polarisationsapparate) gefunden, bei solchen aus der späteren Fötalzeit aber nicht mehr.

2) Siehe Schmidt's Jahrbücher, Bd. 89 , S. 147.

3) Arch. gén., 6. Serie, XXVII, pag. 129 u. 309.

4) Berliner klinische Wochenschrift XII, 21, 1875. 
während des Eindampfens u. s. w. bilde. Während ein bestimmtes Quantum Urin, nach dem angegebenen Verfahren behandelt, keine Reaction gab, zeigte der mit 1--2 Tropfen Chloroform versetzte Harn bei genau gleichem Verfahren deutlich Reduction. Es kam allerdings keine Ausfüllung von rothem Kupféroxydul zu Stande, wohl aber eine Verfärbung der Lösung und ein gelber Niederschlag bei der Abkïhlung der Flüssigkeit.

Solche reducirende Substanzen (im gewöhnlichen Sprachgebrauch, häufig ohne weitere Begründung, Zucker genannt) kommen nun aber ausser béi dem Chloroform noch bei Chloralhydrat, Aetherinhalationen, Curare und anderen Stoffen vor.

Sabarth ${ }^{1}$ ) schreibt speciell dem Aether eine zuckerbildende Wirkung zu und erklärt den Zucker als ein Product der gestörten Respiration. Er recurrirt auf eine Beobachtung Bernards, dass bei Thieren, die Chlorgas zu athmen bekamen, gleichfalls Zucker in den Harn übergehe. Allen diesen Untersuchungen klebt aber der Fehler an, dass nur die Trommer'sche Probe mit dem Urin gemacht, häufig nicht einmal die Harnsäure berïcksichtigt wurde. Theoretisch ist es möglich, dass bei vielen dieser Narcotica wegen einer unvollkommenen Oxydation der Eiweissstoffe die Harnsäure in grösserer Menge gebildet wird und diese vielfach die Hauptursache der Reduction abgiebt. Daneben entstehen wohl noch andere reducirende Stoffe, über welche zur Zeit nichts bekanut ist. Sabarth macht eine Zusammenstellung aller der Stoffe, die, dem Organismus einverleibt, das Auftreten von , Zucker“ im Urin veranlassen.

Eines bleibt wissenschaftlich gesichert: dass die gleiche Substanz im Urin der Kinder auf eine gleiche Wirkung in ihrem Stoffwechsel während der Geburt schliessen lässt, aber in viel geringerem, Grade als bei der Mutter, denn die Kinder kommen nicht narcotisirt zur Welt. Dass trotz der Bewahrheitungen meiner ersten Angaben über den Einfluss der Narcose Kreissender auf den Fötus das Chloroform mit keinen anderen Einschränkungen, als die bisherige Erfahrung lehrt, angewendet werden könne, habe ich schon früher hervorgehoben. ${ }^{2}$ ) Dagegen wird der Nachweis des Chloroforms im Blute meine Auffassung durch-

1) Das Chloroform, Würzburg 1866, S. 192.

2) Siehe Referat der Hamburger Naturforscher-Versammlung, dieses Archiv, Bd. X, S. 401 . 
aus bestätigen, dass eine lang fortgesetzte Chloroformnarcose schliesslich auch das Kind erreichen könne. Ich habe ganz bestimmte Fälle in der Erinnerung, wo bei Eclampsie nach stundenlanger tiefer Chlor oformnarcose, in Fällen, wo die Blase stand, bis unmittelbar zur Zangenoperation, welche rasch und leicht vollendet werden konnte, und wo auch die Anfälle während der Narcose nicht wiedergekehrt waren, doch die Kinder auffallend somnolent geboren wurden. Es ist gezwungen, in solchen Fällen die Kohlensäurenarcose zur Erklärung heranzuziehen, während jeder Geburtshelfer weiss, dass reife unverletzte Kinder unter dem Einflusse der Abkühlung mit einigen kräftigen Athemzügen aus einer tiefen Asphyxie ganz rasch aufleben. Es sind übrigens hierin die Erfahrungen von Schatz') und Benicke mit den meinigen vollkommen übereinstimmend. ${ }^{2}$ )

Es ist erklärlich, dass trotz der so geringen Quantitäten Chloroform, die jeweilen in $30 \mathrm{Gm}$. Blut vorhanden sind, die reducirende Substanz im Urin noch zu finden ist, weil hier das Endproduct der Chloroformwirkung gesammelt wird.

Es fehlte, um einigermassen die Quantität des in den kindlichen Kreislauf eingedrungenen Chloroforms resp. der Salicylsäure zu kennen, vorerst noch an Bestimmungen des Blutgehaltes der Placenta. Budin hat Versuche gemacht, das Blut aus dem Nabelstrange auszudrücken, wenn dieser sofort nach der Geburt des Kindes unterbunden wurde. Schücking hat durch die Waage gezeigt, dass eine ganz erhebliche Quantität Blut dem Kinde entzogen wird, wenn man gegen die alte Hebammenregel sündigt, und vor dem Aufhören des Pulsschlages die Nabelschnur abbindet. Die Demonstration des Blutquantums, welches dem Kinde damit entzogen wird, mit Hülfe der Waage, ist von grosser praktischer Bedeutung.

Ich hatte schon vor langem auf einem anderen Wege das gleiche Ziel angestrebt, nämlich den Blutgehalt in der Placenta $\mathrm{zu}$ bestimmen, wenn regelrecht abgebunden wird, und diesen $\mathrm{zu}$ vergleichen mit demjenigen der zu früh abgebundenen Placenten.

1) Dieses Archiv, Bd. X, S. 402.

2) Ich verweise hier auch auf eine Angabe von Snow, dass die Kinder gegen den Einfluss der äusseren Luft weniger empfindlich seien, und diejenigen von Paul Dubois, dass die Fötalpulse während der Narcose der Mutter an Frequenz zunehmen sollen. 
Es soll hier vorläufig nur Nachricht von Versuchen gegeben werden, welche bei normal abgebundenen Placenten den Blutgehalt bestimmten.

Aus dem Nabelstrange wurde durch leichten Druck ein gewisses Quantum Blut gesammelt, dieses abgewogen, dann die Placenta durch eine Hackmaschine klein gemacht, mit Wasser vollständig ausgelaugt, so lange die Lösung sich noch färbte. Die beiden Blutquantitäten wurden num so fiverdünnt, dass Proben derselben bei gleicherFlüssigkeitsschicht genau dieselbe Farbe hatten. Namentlich bei dem ausgepressten Blute muss man auf eine vollkommene Auflösung der Blutcoagula achten. Wenn die Lösung nicht ganz klar ist, müssen die zum Vergleiche bestimmten Proben filtrirt werden. Durch den einfachen Proportionalsatz kann der Blutgehalt berechnet werden.

1) Normale Geburt, normales Kind. Nabelstrang nach Aufhören des Pulses abgebunden. Placenta wiegt 347, exclusive $22 \mathrm{Gm}$. Blut, welehe durch den Nabelstrang ausgedrückt worden waren. Die Placenta ergab, vollständig ausgezogen, $4050 \mathrm{Ccm}$., und die $22 \mathrm{Gm}$. Blut bei ganz gleicher Verdïnnung 640 Cem., danach berechnete sich dex Gesammtblutgehalt auf 139,2 und $22=161,2 \mathrm{Gm} .=$ $43,69 \%$.

2) Gewicht der Placenta bei gleichen äusseren Verhältnissen wie oben $540 \mathrm{Gm}$; aus dem Nabelstrange gedrückt $20 \mathrm{Gm}$. Bei gleicher Verdünnung ist das Volumensverhältniss $7750: 960$ ergiebt $161,6+20=181,6 \mathrm{Gm}$. Blut $=($ für 560) $32,3 \%$ der Gesammtplacenta.

3) P1acenta (excl. $18 \mathrm{Gm}$. ausgedrüektes Blut) 452 Gramm schwer. Die Flüssigkeitsvolumina verhalten sich wie 6100:480. Daraus berechnet sich ein Blutgehalt von $228+18=256 \mathrm{Gm}$. oder $\mathbf{5} \mathbf{4}, \mathbf{4} \%$ Blut.

4) Placenta 526 inclusive ausgedrücktes Blut 28.

Volumina 7625 : 1500 ergiebt 142,3 Gm. Blut." Hierzu $28=$ 170,3 oder $32 \%$ Blut.

5) Placenta eines siebenmonatlichen Fötus wiegt zusammen incl. $20 \mathrm{Gm}$. Blut, welche nachher ausgedrückt wurden, $348 \mathrm{Gm}$. Die Blutlösungen verhalten sich wie $4750: 1900=50$ oder in toto $70 \mathrm{Gm} .=20,1 \%$ Blut.

Es ist darauf aufmerksam zu machen, dass der Blutgehalt beim unreifen Kinde relativ und absolut viel kleiner gefunden wurde. Die grossen Schwankungen des Durchschnittsgehaltes von $1 / 3-1 / 2$ des Gesammtgewichtes der Placenta zeigen, dass für den Blutgehalt derselben wahrscheinlich verschiedene Finflüsse bestehen. 
Jedenfalls ist der so gefundene Gehalt absolut und relativ überraschend gross ausgefallen. Die Fortsetzung der Versuche und der Vergleich mit den Resultaten, welche Schücking gefunden hat, versprechen ganz interessante Thatsachen zu Tage zu fördern. An der weiteren Ausführung dieser Verhältnisse wird gegenwärtig gearbeitet.

Das Quantum Salicylsäure und Chloroform, das auf den Fötus einwirkt, kann bei diesem grossen Blutgehalte schon nennenswerth sein. Es machen diese Bestimmungen sofort erklärlich, dass trotz der Spuren der Stoffe, welche zuweilen in circa einer Unze Blut gefunden wurden, doch ein Stoffwechselproduct des Chloroforms im Urin der Kinder erscheinen kann.

Eine weitere Frage beim Uebergange des Chloroforms ist die, ob dasselbe zur Entstehung eines Icterus disponire?

Unter den ersten fünf auf die Einwirkung des Chloroforms untersuchten Kindern waren zwei, die im Laufe von drei Tagen einen ausgesprochenen Icterus bekamen. Es war mir damals noch ein weiterer Fall bekannt, bei dem ein Kind mit Icterus gestorben, dessen Mutter während der Geburt chloroformirt worden war.

Nun ist von Leyden im Urin Chloroformirter Gallenfarbstoff gefunden worden. ${ }^{1}$ ) Bekanntlich dient das Chloroform dazu, die rothen Blutkörperchen zu zerstören und das Hämaglobin zur Krystallisation zu bringen, worauf Böttcher in Dorpat ${ }^{2}$ ) zuerst aufmerksam hat. Es liesse sich für einzelne Fälle von Chloroformnarcose annehmen, dass Blutfarbstoff frei werde und wie bei den Hämaglobininjectionen von Tarchan of $f^{3}$ ) der Urin Gallenfarbstoffreaction gebe. Tarchan off selbst hat zwar bei der Chloroformnarcose eines Hundes keinen Gallenfarbstoff in dessen Urin bekommen. Von gleichen Erfahrungen berichtet auch Vogel. 4) Obschon eine Auflösung der Blutkörperchen durch Chloroform ausserhalb des Körpers sicher ist, so ist das Gleiche im Blute des lebenden Organismus noch nicht nachgewiesen.

Ob das Chloroform einen Einfluss auf die Entstehung des Icterus

1) Archiv für klinische Medicin, XII, 1874, S. 32.

2) Blutkrystalle, Dorpat 1862.

3) Archiv für Physiologie von Pflüger, Bd. IX, S. 62.

4) Zur Theorie des Icterus. 45. Naturforscher-Versammlung in Leipzig. S. 75 . 
habe, konnte nur die klinische Statistik beweisen. Die dahingehenden Beobachtungen wurden in den Jahren 1874 und 1875 in der Klinik des Herrn Prof. Gusserow in Strassburg gemacht. Es kamen nach Anwendung von Chloroform 22 Mal Icterusfälle vor, $16 \mathrm{Mal}$ ohne Chloroform. Unter 31 Kindern, die unter dem Einflusse der Narcose geboren worden, trat 22 Mal eine deutliche Gelbfärbung ein, $9 \mathrm{Mal}$ nicht. Es repräsentirt dies ein Zahlenverhältniss von etwas über $2: 1$. Danach hätten von den 31 darauf beobachteten Kindern etwas über zwei Drittel Icterus bekommen. Weil aber $\mathrm{Kehrer}^{1}$ ) für 690 in Wien beobachtete Kinder in $68,7 \%$ Icterus verzeichnet fand, beweist das oben erwähnte Zahlenverhältniss, dass das Chlor oform keine ätiologische Bedeutung für den Icterus neonatorum habe. Keines der nach Chloroformgebrauch icterisch gewordenen Kinder ist der Gelbsucht erlegen. Das einzige, welches starb, zeigte bei der Section einen undurchgängigen Ductus choledochus.

Bei der grossen Häufigkeit des Icterus neonatorum ist es von vornherein unwahrscheinlich, dass derselbe jeweilen ein katarrhalischer sei. Der gutartige und rasche Verlauf spricht entschieden dagegen. Die gleiche. Thatsache lässt auch den Gedanken an einen pyämischen Ursprung des Icterus neonatorum, welche Deutung auch schon versucht worden ist, für die grosse Mehrzahl der Fälle nicht aufkommen. Es kann sich also nur noch um einen Resorptions-oder einen hämatogenen Icterus handeln. Nach Morgagni, Autenrieth und Frerichs soll durch Verminderung des Blutdruckes in der Pfortader bei Beginn des Lungenkreislaufes eine Resorption von Galle aus den Gallenkanälchen ins Blut stattfinden. Es verursacht das Einschalten des Lungenkreislaufes mit Wahrscheinlichkeit eine kurz vorübergehende Druckherabsetzung, aber andererseits wächst auch durch die gleichzeitige Ausschaltung des Placentarkreislaufes der Blutdruck beim Neugeborenen sehr stark an. Die Vermehrung des Druckes trifft namentlich diejenigen arteriellen Gefässgebiete, die von den abgesperrten Nabelarterien rückwärts zunächst liegen. Wahrscheinlich wird sehr rasch in Folge des höheren arteriellen Druckes auch die Druckverminderung in der Pfortader ausgeglichen. Wenn dann auch wirklich eine Resorption von Galle stattgefunden hat, so muss doch kurze Zeit nachher bei der Ausgleichung der Druckverhält-

1) Oesterreichisches Jahrbuch für Pädiatrik, Jahrgang-1871, II. Bd., S. 73. 
nisse, der übergetretene Gallenfarbstoff von der Leber wieder aus dem Blute abgeschieden werden. Es ist darum sehr wenig plausibel, dass diese ganz rasch verlaufende Druckschwankung zur Entstehung eines Resorptionsicterus genüge.

Nur in den Fällen, wo durch $\mathrm{zu}$ frühes Unterbinden dès Nabelstranges dem Kinde viel Blut abgesperrt wird, kann das Sinken des Blutdruckes so stark und anhaltend sein, dass dabei an eine Resorption von Gallenfarbstoff zu denken ist.

So weit ich die Literatur durchsehen konnte, ist bei der Mehrzahl icterischer Neugeborener, so oft darauf untersucht wurde, kein Gallenfarbstoff im Urin gefunden worden. Es ist dies eine Thatsache, die für die Annahme eines hämatogenen, aber ganz besonders gegen einen Stauungsicterus spricht.

$\mathrm{Zu}$ berïcksichtigen ist ein Befund, den $\mathrm{Orth}^{\mathbf{1}}$ ) bei Icterus neonatorum machen konnte. In verschiedenen Geweben und in der Haut kamen selbst bei den gowöhnlichen Fällen von Gelbsucht der Neugeborenen Bilirubinkrystalle vor, beim Icterus der Erwachsenen aber nur in den schwersten Formen der Krankheit. Zwei Mal war auch der Grund und der Hof von Magengeschwüren mit den gleichen Krystallen angefüllt. Von anderer Seite wurden dieselben für Hämatoidinkrystalle, also ein Umsetzungsproduct des Blutfarbstoffes gehalten. Es ist höchst wahrscheinlich, dass Bilirubin und Hämatoidin identische Farbstoffe sind, dass also der Blutfarbstoff ausserhalb der Leber in Gallenfarbstoff übergehen kann. Tarchan off's ${ }^{2}$ ) Injectionen von Blutfarbstoff und von Wasser in die Jugularis eines Hundes führten eine sehr bedeutende Vermehrung des Farbstoffes in der durch eine Fistel aufgefangenen Galle herbei. Das Gleiche macht eine Bilirubininjection. Niemals war aber bei diesen Versuchen Gallenfarbstoff im Urin zu finden. Daraus schliesst der genannte Forscher, dass der Farbstoff durch die Leber in gleich energischer Weise aus dem Blute abgeschieden wird wie die Gallensäuren, so lange die Gallenausscheidung nicht gehemmt ist.

Diese Thatsachen führen zu der Auffassung, dass der Icterus neonatorum einem Austritt von Blut aus den Gefässen der Haut, dem Zerfallen der Blutkörperchen

1) Virchow's Archiv, Bd. 63, 1875.

2) Pflüger's Archiv, Bd. IX, S. 329 u. ff. 
und dem Freiwerden des Blutfarbstoffes seine Entstehung verdankt.

Wenn man berücksichtigt, welchen Einflüssen die Haut eines. Fötus während der Geburt ausgesetzt wird, so sind capilläre Blutaustritte unter die Haut recht gut möglich. Beim Durchgange durch die Geburtswege erleidet die gesammte Körperoberfläche einen sehr erheblichen Druck, welcher mit der Ausstossung der Frucht plötzlich aufhört. Es muss der Zufluss zu der Haut mit einem Schlag viel stärker sein, und es ist die rosa-, oft sogar krebsrothe Färbung der Haut unmittelbar nach der Geburt allgemein bekannt.

Die capillären Blutextravasate müssen ceteris paribus um so eher eintreten, wenn die Hautreizungen heftiger waren, wozu eine forcirte Reinigung der Haut durch die Hebammen auch das ihrige beitragen kann; es werden dieselben eher zu erwarten sein bei den Kindern Erstgebärender, wo die Reibungen und der Druck der Genitalien stärker sind als bei Mehrgebärenden, aus dem gleichen Grunde ebenfalls eher bei etwas grösseren Kindern. Bei asphyktischen und zu früh Geborenen spielt die mangelhafte Entfaltung der Lungen und die häufiger eintretende Atelectase eine Rolle, indem dadurch eine Staung im Venensysteme entsteht, die sich schon durch die bläuliche Färbung der Haut oft bemerkbar macht. Ferner zeichnet sich gerade die Haut der zu früh Geborenen unmittelbar nach der Geburt meistens durch eine auffallend rothe Farbe aus. Es wird diese Theorie nicht alle Fälle von Icterus neonatorum erklären, aber mit Wahrscheinlichkeit. die Mehrzahl derselben. Die Richtigkeit wird sich durch Fortsetzung der Orth'schen Beobachtungen erweisen lassen.

In der verdienstvollen Arbeit von Kehrer ist durch directe Beobachtung nachgewiesen, dass der Icterus neonatorum bei Knaben um 6,6\% häufiger sei als bei Mädchen, dass sich zusammengehörige Zwillinge bald gleich, bald ungleich in Bezug auf Icterus. verhalten, dass er um 4,2\% häufiger sei bei den Kindern Erstals bei denen Mehrgebärender, um 17,7\% häufiger bei zu früh geborenen Kindern, viel häufiger bei Beckenendlagen, bei Geburten bis zu 6 Stunden in $62,2 \%$, bis zu 12 Stunden in $74,1 \%$, bis zu 30 Stunden in $74,6 \%$ und bei Geburten über 30 Stunden, die aber nur noch $11,3 \%$ aller Geburten betragen in $66,6 \%$. Eine spätere Ausscheidung von Meconium hat auf die Entstehung: eines Icterus neonatorum keinen Einfluss (Widerlegung der Theorie 
von P. Frank, dass der Icterus neonatorum durch die Resorption der Gallenfarbstoffe des Meconiums zu 'Stande komme). Sowohl bei den Sectionen wie bei den Untersuchungen lebender icterischer Kinder waren die Faeces stets dottergelb, nie blass oder lettig gefärbt, wie schon Burdach wusste und Leyden bestätigte. Es kann darum in allen diesen Fällen kein Stauungsicterus gewesen sein. Diese Angaben Kehrer's erhöhen die Wahrscheinlichkeit der oben gegebenen Theorie wesentlich.

Ein französischer Autor, Poncet ${ }^{1}$ ), sah bei vielfachen und ausgedehnten Verletzungen mit Blutaustritt unter die Haut eine allgemeine icterische Verfärbung eintreten, ohne Gallenfarbstoffausscheidung im Urin.

Er benennt dies als traumatischen Hämato-Icterus und hält das ausgetretene Blut für das Farbstoffdepot. Andral hatte schon früher die Auffassung vertreten, dass gewisse Fälle von Icterus (ohne specielle Beziehung auf den Icterus neonatorum) von Ecchymosen im Rete Malpighi herrühren:

Wegen dieser Verhältnisse kann es nun leicht kommen, dass gerade bei Geburten, bei welchen schliesslich Chloroform verwendet werden muss, das Kind einige Tage später einen Icterus bekommt. Nach den oben angegebenen Zahlenverhältnissen ist aber trotzdem das Chloroform als ätiologisches Moment auszuschliessen.

Für die kreissenden Frauen ist die Anwendung der Chloroformnarcose eine grosse Wohlthat und oft für den Arzt eine nicht zu unterschätzende Erleichterung bei Operationen. Ich habe auch aus diesen Gründen schon oben betont, dass meine Auffassung nicht dahin gehe, wegen des Chloroformnachweises, dessen Gebrauch einzuschränken. Es lässt sich daraus nur der Schluss ziehen, dass man bei langer Chloroformnarcose auch auf das Kind einige Rücksicht nehmen müsse.

Kreissende werden gewöhnlich mit auffallend geringen Quantitäten Chloroform narcotisirt, bekommen häufig gar kein Brechen danach und bleiben von den übrigen unangenehmen Nachwirkungen fast vollständig verschont. Dagegen ist als Nachtheil der Narcose die schon von Scanzoni ${ }^{2}$ ) hervorgehobene Begünstigung

1) De l'ictère hématique traumatique, Paris 1874.

2) Beiträge zur Geburtshülfe und Gynäkologie, II. Band, 1855. 
von Atonie des Uterus in der Nachgeburtsperiode öfters bestätigt worden.

Der Engländer Sansom, welcher zu den grössten Verehrern des Chloroforms zu zählen ist, hat doch den Eindruck nicht von der Hand weisen können, dass der Geburtsverlauf etwas verzögert werde. Für die geringen Grade der Narcose, bei welcher die Reflexe von Seiten der Conjunctiva noch nicht aufgehoben sind. wird die Uterusthätigkeit kaum afficirt - es fällt für die Geburt höchstens die Action der Bauchpresse weg. In den meisten Fällen wird damit die Geburt eher befördert als verzögert, denn das stete Mitpressen bei den Schmerzen ruft gewöhnlich nur eine unregelmässige Wehenthätigkeit und eine baldige Erschöpfung der Kreissenden hervor. In den ausgesprochensten Fällen von Wehenstörung, den Krampfwehen, leistet das Chloroform bekanntlich vorzügliche Dienste, und wohl wesentlich nur durch die Verhinderung des andauernden Pressens und die Regulirung der Wehen. Es sind Fälle zu beobachten, wo die krampfhafte Contraction des Muttermundes - die sogenannte Strictur - fehlt, und also von dieser Seite dem Vorrücken des Kopfes kein Hinderniss entgegensteht, und trotzdem die kräftigsten austreibenden Wehen kein Vorwärtsrücken des Kopfes zu Stande bringen. Hier hilft sehr oft eine kleine Ruhepause durch die Chloroformnarcose, und in kurzer Zeit fühlt man den Kopf trotz der scheinbaren Ruhe und Wehenlosigkeit tiefer in dem Becken stehend.

In Beziehung auf die Atonie des Uterus und die dadurch bedingten Nachblutungen ist auch entgegenzuhalten, dass in vielen solchen Fällen die zu rasche Entleerung des Uterus dieselben eher verschuldet als das Chloroform. Bei Zangenoperationen wegen Wehenschwäche kann ein Ansaugen respective eine Druckverminderung in der Uterinhöhle entstehen, wenn die Gebärmutter schlaff bleibt und das Kind nicht mehr umschliesst. Es wird sicher dadurch eine Trennung der Placenta von ihrer Haftfläche begünstigt und eine Disposition für Blutungen in der Nachgeburtsperiode geschaffen. Es kann in dieser Beziehung nicht zu viel darauf aufmerksam gemacht werden, dass in der letzten Periode einer künstlichen Entbindung durch Druck auf den Fundus uteri stets energische Contractionen der Gebärmutter eingeleitet werden müssen, um die atonischen Blutungen zu vermeiden.

Dass das Entstehen einer Atonie des Uterus durch eine vorangegangene Chloroformnarcose noch begünstigt werde, ist nach 
den Untersuchungen Winckel's als sicher anzunehmen. Beim Kaiserschnitt macht sich die Energielosigkeit am unangenehmsten geltend, und diese Erfahrung wurde bei der in hiesiger Klinik ausgeführten Operation ebenfalls gemacht. Es ist für solche Fälle zu rathen, vor Ausführung der Sectio caesarea nicht zu chloroformiren.

Die klinische Seite der Chloroformnarcose ist übrigens von vielen Gynäkologen (Scanzoni, Spiegelberg, Martin, Germann, Winckel, Grenser, Siebold, Hohl, Sansom, Ellis u. s. w.) so erschöpfend behandelt worden, dass ich dieselbe nicht weiter berühren will. 